\title{
Spinal Cord Editor's Page June 2007
}

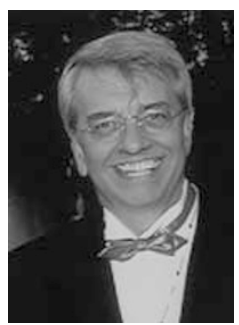

This issue contains eight original contributions related to management in patients with spinal cord lesion (SCL).

In 'Spinal Cord Injuries; comprehensive management and research' (Blackwell Scientific Publications 1973), Sir Ludwig Guttmann reflects upon the ultimate integration of the spinal man, from which I take the following ideas. There is a wealth of talent lying dormant in human beings and, ironically, it often takes a disaster in life resulting in severe disability to bring it out. The essence of the rehabilitation of paraplegics and tetraplegics lies in restoring activity of the mind, promoting their determination to utilize those talents that are already apparent and encouraging new talents by awakening and mobilizing the intellectual potentialities that are still dormant. When the disabled develop their dormant talents with determination and concentrated effort, they are rewarded with pride and satisfaction and the community is enriched with priceless treasures.

Although written more than 30 years ago, this book is still very up to date. In the rehabilitation and reintegration process, early education and training of the disabled child and retraining of the adult are the indispensable essentials. One can only wish that the community at large will also recognize new talents in the field of training and employment. The old prejudice toward a person in a wheelchair dies hard. Continuous education of the public is needed and supporting caregivers is mandatory. This means that information and education must be actively and broadly provided. There is no one rule for this, as it will depend on the patient, the caregivers and the community.

One study published in this issue focuses special attention on those who care for patients with SCLs (Blanes and co-workers). This assessment of the health-related quality of life (HRQoL) of primary caregivers of persons with paraplegia due to traumatic spinal cord injury (SCI) not only shows the hours per day needed for this care, but also the impact on caregivers' quality of life. Caregivers are indispensable in this management and thus deserve a lot of attention.

A study by Pagliacci and co-workers reports on readmissions and major complications commonly seen after SCI at 7 spinal units and 17 rehabilitation centers. The authors show the failure of which functions on discharge was the most common predictive factor of poor outcome.

Manns and May present a study of eight focus groups, some with community dwelling people with SCI and others with unpaid caregivers such as family and friends. The participants discuss factors that help and hinder their long-term health. The five categories in which those factors are captured are identified and a framework that illustrates links between categories was constructed.

Education and training must be implemented at an early stage in the wake of severe disability to counteract the adverse psychological effects that almost inevitably follow: frustration, apathy and resignation. Elfström and co-workers evaluate coping strategies employed to manage the consequences of an SCL, which were distinctly related to emotional well-being. They evaluated the 'Acceptance coping scale', the 'Fighting spirit scale' and the 'Social reliance scale' and propose potential improvements.

Murray and co-workers examine the patient's perspective of the impact of SCI on physical, cognitive and emotional function and quality of life in Australia. Stanford and co-workers present an interesting study on mental health and community placement outcomes for the survivors of SCL suicide attempts.

Planning rehabilitation greatly depends on a proper diagnosis of the extent of the injury, which is also needed to compare studies. Two studies on evaluation methods are published in this issue. 
Savic and co-workers study levels of agreement in ASIA clinical examinations between two experienced examiners. They determine if an established degree of variability due to inter-rater differences should be taken into account in the design of clinical trials with more than one assessor.

Mulcahey and co-workers evaluated the ISCSCI (International Standards for Neurological Classification of Spinal Cord Injury) examinations in children of different ages. They report on recommended values for motor and sensory examinations.

One book review and a correspondence between Dr Silver and Dr Wilsmore are included. These letters deal with the original manuscript of thermoregulation in tetraplegic patients.

I wish you all much joy in reading these contributions

Spinal Cord (2007) 45, 397-398; doi:10.1038/sj.sc.3102082

J-J Wyndaele

Editor

E-mail: spinalcord@uza.de 